\title{
COVID-19 "La Piel Se Expresa” Comportamiento en la Población Pediátrica
}

COVID-19 "The skin is expresses" Behavior in the Pediatric Population

Héctor Rubén Caballero Castro*, Melida de Jesús

Galeas Oliva**, María Fernanda Díaz Guifarro*

\section{RESUMEN}

La situación actual en Honduras y a nivel mundial ha cambiado drásticamente con la aparición de un nuevo tipo de coronavirus que produce el Síndrome Respiratorio Agudo Severo (SARSCoV-2) (Covid 19). Lo que inicialmente se creía causaba sintomatología similar a un simple resfriado u otras patologías respiratorias virales comunes, ha evolucionado y ocasionado daños catastróficos con innumerables pérdidas humanas y económicas. Muchas han sido las formas de presentación del reciente coronavirus, el cual ha demostrado en múltiples reportes de casos a nivel mundial que ataca diferentes sistemas; como ser el gastrointestinal, respiratorio, cardíaco y cutáneo en los diferentes grupos etarios.

No obstante, el cuadro que aparece en algunos casos de población pediátrica es mucho menos severo, con manifestaciones muy leves como rinorrea, congestión nasal, fatiga, fiebre, tos, entre otros.(1) También hay reportes de casos de COVID-19 positivo que inicialmente manifestaron una erupción cutánea sin síntomas respiratorios; éstos cuadros cutáneos podrían ser el preludio de un paciente infectado y ante ésta sospecha, se puede prevenir la transmisión de la enfermedad; así冈 como también establecer el diagnóstico y tratamiento oportuno.

\section{PALABRAS CLAVE}

Niños, Coronavirus, Kawasaki-like, Covid-19, Exantema

\footnotetext{
*Pediatra Dermatólogo IHSS Regional del Norte

**Pediatra en Emergencia de IHSS Regional del Norte

* Doctora en Medicina y Cirugía (Pasante Dermatología

Pediátrica en IHSS Regional del Norte)

Dirigir correspondencia a: h_caballerocastro@hotmail. com, maferdiaz1992@gmail.com

Recibido: 20 de Agosto de 2020 Aceptado: 20 de Sep-

tiembre de 2020
}

\begin{abstract}
The current situation in Honduras and worldwide has changed dramatically with the appearance of a new type of coronavirus that produces Severe Acute Respiratory Syndrome (SARS-CoV-2) (Covid 19). What was initially believed to cause symptoms similar to a simple cold or other common viral respiratory diseases, has evolved and caused catastrophic damage with innumerable human and economic losses. There have been many forms of presentation of the recent coronavirus, which has shown in multiple case reports worldwide that it attacks different systems, such as the gastrointestinal, respiratory, cardiac and skin systems in different age groups.
\end{abstract}

However, the condition that appears in some cases in the pediatric population is much less severe, with very mild manifestations such as rhinorrhea, nasal congestion, fatigue, fever, cough, among others. (1) There are also reports of positive COVID-19 cases who initially manifested a skin rash without respiratory symptoms; these cutaneous pictures could be the prelude to an infected patient and in view of this suspicion, the transmission of the disease can be prevented, as well as establishing the diagnosis and timely treatment.

\section{KEYWORDS}

Children, Coronavirus, Kawasaki-like, Covid-19, Rash.

\section{INTRODUCCIÓN}

El SARS-CoV-2 se ha extendido rápidamente hasta alcanzar el nivel de una enfermedad pandémica. La infección por el nuevo coronavirus identificado en el año 2019 en Wuhan (China), puede afectar diferentes sistemas, incluyendo el 
órgano más grande del cuerpo, la piel. Hay pocas descripciones de las manifestaciones cutáneas de COVID-19. Al inicio del brote, la afectación en pacientes pediátricos fue descrita sólo excepcionalmente, pero con la extensión progresiva del virus, cada vez se han reportado más casos en niños, siendo la mayor parte de ellos leves o asintomáticos. Estos pacientes presentan pronta recuperación en períodos de 1-2 semanas, son muy pocos los casos de severidad. ${ }^{(1,2)}$ Esta información puede resultar útil para manejar y reconocer pacientes con poca sintomatología y puede proporcionar información pronóstica, asimismo ser de utilidad en el área epidemiológica, especialmente en lugares donde las pruebas de diagnóstico laboratorial son escasas. ${ }^{(3,4)}$

\section{GENERALIDADES}

Ciertos tipos de manifestaciones cutáneas se han hecho evidentes con ésta pandemia. Siendo el coronavirus un virus de la familia RNA o ARN, puede dar manifestaciones cutáneas diversas, acompañado o no de fiebre, síntomas gastrointestinales, respiratorios y cardíacos.

Los patrones de presentación dermatofisiopatológicos vistos en el Instituto Hondureño de Seguridad Social (IHSS) Regional del Norte, fueron: (Ver Figura 1)

1. Exantemas virales ( como respuesta inmunológica a nucleótidos virales)

a. Rash maculopapular

\section{Forma petequial y vasculítica}

a.Kawasaki clásico o típico

b. Kawasaki-like

c. Síndrome Multisistémico inflamatorio pe diátrico

3. Erupciones dermatológicas secundarias a consecuencias sistémicas causadas por el COVID-19 y/o a interacción y reacciones adversas a medicamentos.

Las manifestaciones más severas de tipo Síndrome Steven Johnson o Necrólisis Epidérmica Tó- xica, fueron más vistas en lactantes. Cabe mencionar que dentro de los pacientes ingresados en el IHSS dos eran lactantes, uno de 2 meses de edad y otro de 3 meses de edad que estuvieron en la Unidad de Cuidados Intensivos Pediátricos (UCIP) con Necrólisis Epidérmica Tóxica.

Figura No.1: Patrones dermatológicos observados en el Instituto Hondureño de Seguridad Social Regional del Norte.

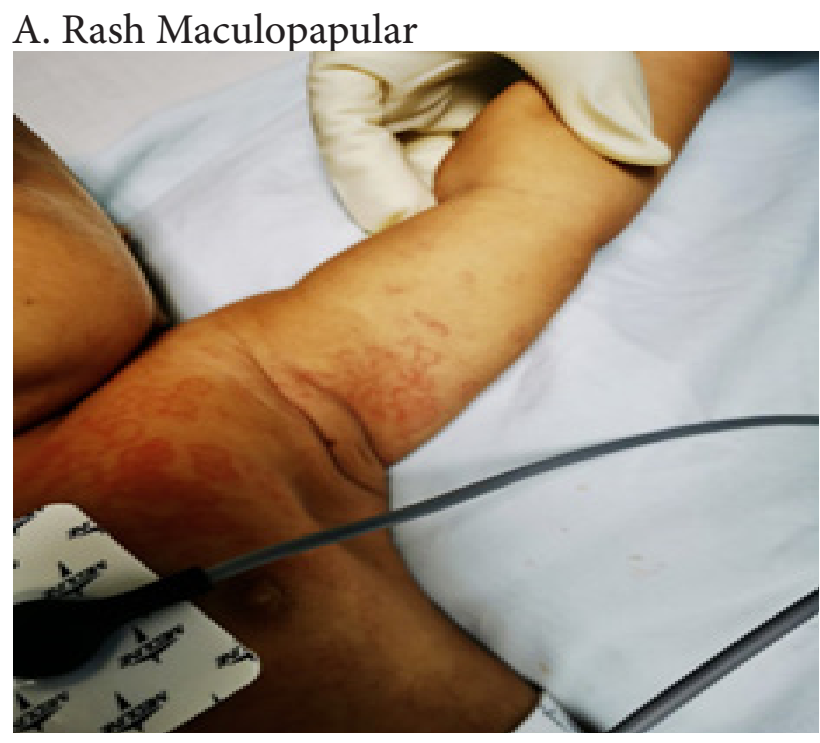

B. Lesiones Petequiales y Vasculíticas.

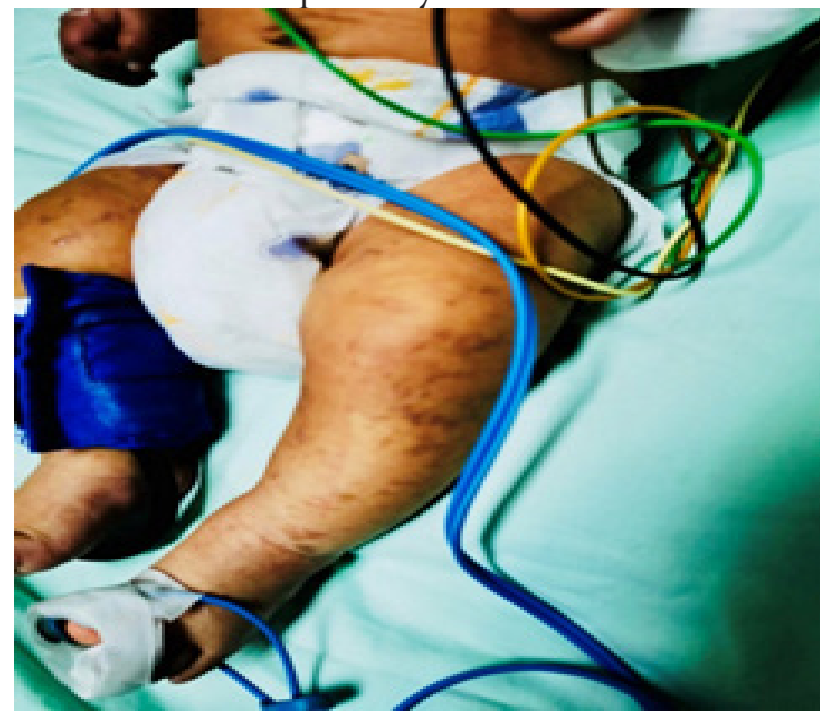


C. Erupciones Dermatológicas secundarias

a reacciones medicamentosas (Necrólisis Epidérmica Tóxica).

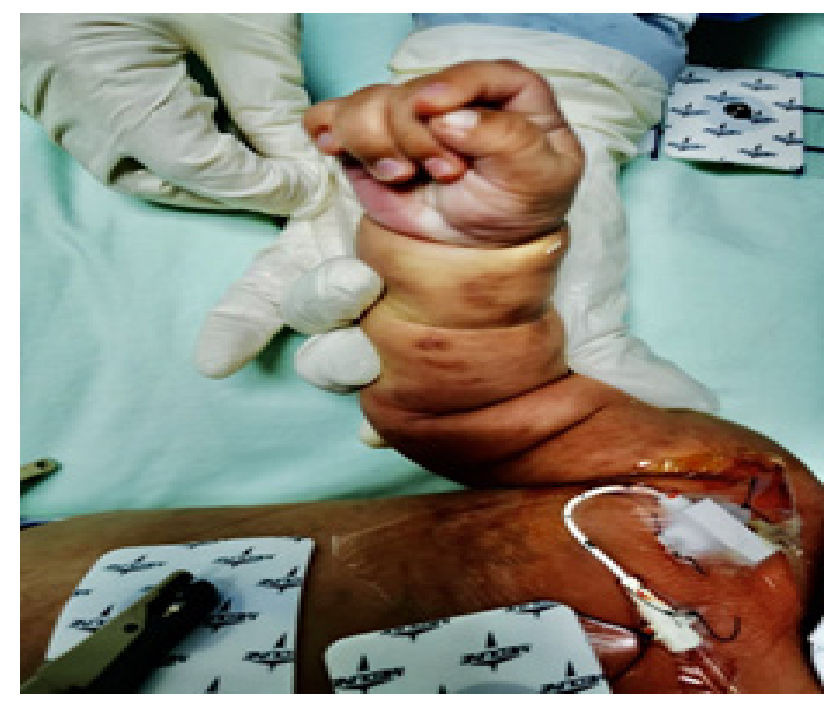

D. Inyección conjuntival y lengua en fresa de la Enfermedad de Kawasaki.

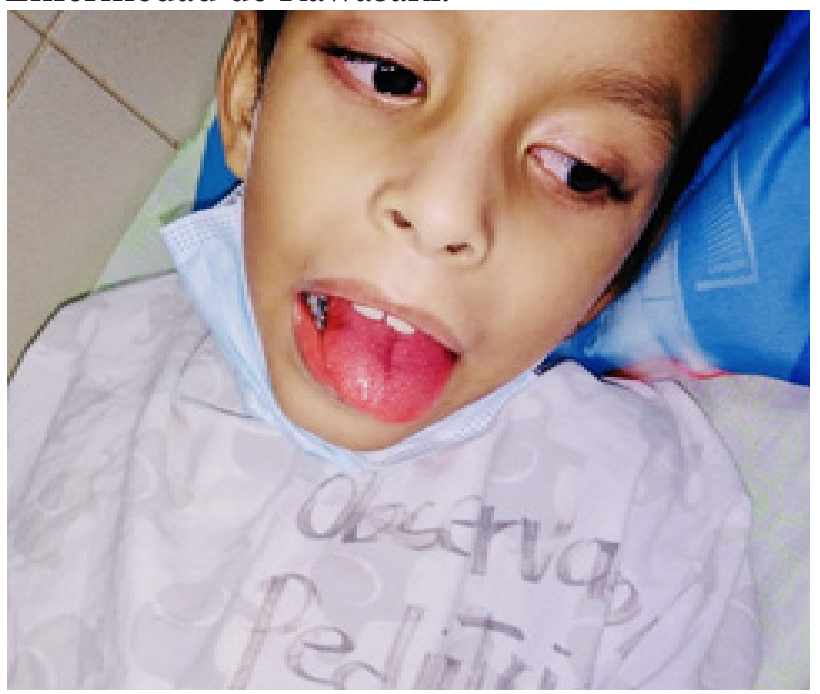

Los pseudosabañones, los cuales no hemos observado hasta la fecha a diferencia de otros estudios realizados en otros países, se presentan más en pacientes jóvenes, asociado a COVID-19 moderado. ${ }^{(5-7)}$ En el Instituto Hondureño de Seguridad Social Regional del Norte, por la experiencia en pacientes pediátricos atendidos hemos observado la presencia de patrones cutáneos en un $5 \%$ de la población pediátrica.

Estos casos no pueden excluir la presencia absoluta de Covid-19 en los pacientes, debido a que la infección tiene diferentes comportamientos en cada organismo y en población pediátrica puede ser indicativo que el paciente tuvo la enfermedad pero la respuesta inmediata de su sistema inmune más enérgico o un aparato respiratorio más saludable, la haya eliminado satisfactoriamente. (8) A su vez, en algunos países de Europa y ciudades en los Estados Unidos ${ }^{(9)}$, se han reportado otros casos de SARS Cov-2 en niños, relacionado con otras patologías vinculadas a la piel, como el síndrome de Kawasaki o con un síndrome Kawasaki-like y el denominado Síndrome Inflamatorio Multisistémico Pediátrico (PMIS-TS) por sus siglas en inglés, "Pediatric Multisystem Inflammatory Syndrome temporally associated with SARSCoV-2", el cual todavía se está estudiando y actualizando a nivel mundial. En los pacientes pediátricos reportados en éstos países, se encontró en sus hallazgos de pruebas laboratoriales con marcadores inflamatorios elevados, linfopenia, trombocitopenia y neutrofilia, así como la presencia de algunas manifestaciones comúnmente descritas en el Síndrome de Kawasaki como fiebre,rash,conjuntivitis y edema en manos y pies. ${ }^{(9,10)}$ En el IHSS Regional del Norte son pocos los casos reportados con dichas similitudes, encontrándose dos casos de pacientes con Enfermedad de Kawasaki clásico, los cuales presentaron aneurismas coronarios, tres pacientes con enfermedad Kawasakilike y otros tres con Síndrome Inflamatorio Multisistémico. Cabe mencionar que a todos los pacientes ingresados en la emergencia pediátrica, sin importar el motivo de consulta, se les realiza prueba rápida para Covid-19 y al captar un caso positivo, se procede a realizar rt-PCR, el cual posteriormente se maneja de acuerdo a los protocolos intrahospitalarios. Podemos concluir que, las manifestaciones dermatológicas descritas asociadas al nuevo SARS-CoV-2, son muy similares a las presentadas en otras patologías de origen viral y podrían ser las manifestaciones iniciales de la infección, en algunos casos asintomáticos. Por lo tanto, es de interés médico la detección temprana de éstas lesiones a fin de evitar complicaciones futuras. (6,11) Se espera que en el futuro cercano, podamos fortalecer 
los sistemas de salud, para evaluar y vigilar más eficazmente los casos sospechosos de SARSCoV-2, a fin de detectar más tempranamente la enfermedad para controlar su propagación en las comunidades. $^{(12)}$

\section{REFERENCIAS BIBLIOGRÁFICAS}

1. (PDF) Cutaneous Manifestations in Children with COVID-19 [Internet]. [cited 2020 Aug 3]. Available from: https://www.researchgate. net/publication/341901310_Cutaneous_ Manifestations_in_Children_with_COVID-19

2. Hong H, Wang Y, Chung HT, Chen CJ. Clinical characteristics of novel coronavirus disease 2019 (COVID-19) in newborns, infants and children [Internet]. Vol. 61, Pediatrics and Neonatology. Elsevier (Singapore) Pte Ltd; 2020 [cited 2020 Aug 3]. p. 131-2. Available from: https://pubmed.ncbi.nlm.nih.gov/32199864/

3. Singhal T. A Review of Coronavirus Disease-2019 (COVID-19) [Internet]. Vol. 87, Indian Journal of Pediatrics. Springer; 2020 [cited 2020 Aug 3]. p. 281-6. Available from:https:// pubmed.ncbi.nlm.nih.gov/32166607/

4. Recalcati S. Cutaneous manifestations in COVID-19: a first perspective. Vol. 34, Journal of the European Academy of Dermatology and Venereology. Blackwell Publishing Ltd; 2020. p. e212-3.

5. Cordoro KM, Reynolds SD, Wattier R, McCalmont TH. Clustered cases of acral perniosis: Clinical features, histopathology, and relationship to COVID-19.

Pediatr Dermatol [Internet].

2020 May 1 [cited 2020 Aug 3];37(3):419-23. Available from:https://pubmed.ncbi.nlm.nih. gov/32396999/

6. Andina D, Noguera-Morel L, BascuasArribas M, Gaitero-Tristán J, Alonso-Cadenas JA, Escalada-Pellitero S, et al. Chilblains in children in the setting of COVID-19 pandemic.
Pediatr Dermatol [Internet]. 2020 May 1 [cited 2020 Aug 3];37(3):406-11. Available from: https://pubmed.ncbi.nlm.nih.gov/32386460/

7. El Hachem M, Diociaiuti A, Concato C, Carsetti R, Carnevale C, Ciofi Degli Atti M, et al. A clinical, histopathological and laboratory study of 19 consecutive Italian paediatric patients with chilblain-like lesions: lights and shadows on the relationship with COVID-19 infection. J Eur Acad Dermatology Venereol [Internet]. 2020 [cited 2020 Aug 3]; Available from: https:// pubmed.ncbi.nlm.nih.gov/32474947/

8. Mastrolonardo M, Romita P, Bonifazi E, Giuffrida R, Lotti T, Foti C, et al. The management of the outbreak of acral skin manifestations in asymptomatic children during COVID-19 era [Internet]. Dermatologic Therapy. Blackwell Publishing Inc.; 2020 [cited 2020 Aug 3]. Available from: https://pubmed.ncbi.nlm.nih. gov/32424886/

9. Panupattanapong S, Brooks EB. New spectrum of COVID-19 manifestations in children: Kawasaki-like syndrome and hyperinflammatory response. Cleve Clin J Med. 2020 Jun 3;

10. Jones VG, Mills M, Suarez D, Hogan CA, Yeh D, Bradley Segal J, et al. COVID-19 and Kawasaki Disease: Novel Virus and Novel Case. Hosp Pediatr [Internet]. 2020 Apr 7 [cited 2020 Jul 22];10(6). Available from: https://pubmed. ncbi.nlm.nih.gov/32265235/

11. Colonna C, Monzani NA, Rocchi A, Gianotti R, Boggio F, Gelmetti C. Chilblainlike lesions in children following suspected COVID-19 infection. Pediatr Dermatol [Internet]. 2020 May 1 [cited 2020 Aug 3];37(3):437-40. Available from: https://pubmed. ncbi.nlm.nih.gov/32374033/

12. Rodriguez-Morales AJ, Sánchez-Duque JA, Botero SH, Pérez-Díaz CE, Villamil-Gómez WE, Méndez CA, et al. Preparation and control 
of the coronavirus disease 2019 (COVID-19) in Latin America. ACTA MEDICA Peru [Internet]. 2020 Mar 18 [cited 2020 Aug 6];37(1):3-7. Available from: http://www.amp.cmp.org.pe/ index.php/AMP/article/view/909 